\title{
Neuronal Activity in the Medial Prefrontal Cortex during Pavlovian Eyeblink and Nictitating Membrane Conditioning
}

\author{
Donald A. Powell,1,2,3 Brian Maxwell, ${ }^{1,2}$ and James Penney ${ }^{1}$ \\ ${ }^{1}$ Neuroscience Laboratory, Veterans Affairs Medical Center, Columbia, South Carolina 29201, 2Department of \\ Psychology, University of South Carolina, Columbia, South Carolina 29208, and ${ }^{3}$ Department of Psychiatry and \\ Behavioral Science, University of South Carolina School of Medicine, Columbia, South Carolina 29208
}

The present study assessed Pavlovian eyeblink (EB) conditioning, using tones and periorbital shock as the conditioned and unconditioned stimuli (CS and US), and nictitating membrane (NM) conditioning, using tones and airpuffs as the CS and US. During each experiment, CS-evoked changes in multiple-unit activity (MUA) in the medial prefrontal cortex (mPFC) were recorded. Concomitant heart rate $(\mathrm{HR})$ conditioned responses (CRs) were also recorded. A nonassociative control group received explicitly unpaired presentations of the CS and US in each experiment. Increases in both NM and EB CRs occurred over sessions in the paired, but not the unpaired, groups. Decelerative HR CRs also occurred in the eyeshock, but not the airpuff, group. Although tone-evoked increases in neuronal activity were obtained during 10 initial tone-alone presentations in all groups, this activity habituated over trials. CS-evoked in- creases in neuronal activity also occurred, but this activity was considerably greater in the group that received periorbital shock as the US. During subsequent extinction trials, decreases in tone-evoked neuronal activity occurred in this group, compared with the previous CS/US paired trials. CS-evoked MUA increases were minimal during all except the pretraining phase of the study in the CS/US unpaired control groups and in the paired airpuff group. These findings show that neuronal activity during associative learning occurs in the MPFC during Pavlovian EB, as well as HR conditioning, but this activity apparently reflects an affective component to learning that is only indirectly related to skeletal conditioning.

Key words: heart rate; autonomic conditioning; pseudoconditioning; multiple-unit activity; electrophysiology; rabbits
It has been shown in previous experiments that the medial prefrontal cortex (mPFC) is intimately involved in the learned cardiac decelerations obtained during classical (Pavlovian) heart rate (HR) conditioning (Buchanan and Powell, 1993). The unconditioned stimulus (US) in these experiments in most cases consisted of periorbital shock. However, the relatively long CS/US intervals [i.e., interstimulus intervals (ISIs)] that are optimal for autonomic conditioning do not support eyeblink (EB) or nictitating membrane (NM) conditioning, which normally also occurs to tone/ eyeshock contingencies with brief ISIs (Buchanan and Powell, 1993). The optimum ISI for eliciting EB conditioning is 300-500 msec (Schneiderman and Gormezano, 1964), whereas the ISI that elicits the largest magnitude HR conditioned responses (CRs) is 4-6 sec (VanDercar and Schneiderman, 1967; Kazis et al., 1973; Powell et al., 1974). Using this relatively long and optimum ISI for HR conditioning, it has been shown that lesions of the mPFC greatly diminish the magnitude of the HR CR in both rabbits (Buchanan and Powell, 1982) and rats (Frysztak and Neafsey,

Received March 11, 1996; revised June 20, 1996; accepted July 15, 1996.

This research was supported by Department of Veterans Affairs Institutional Research Funds awarded to the William Jennings Bryan Dorn Veterans Affairs Medical Center, Columbia, SC. We thank Judy Burris and Andrew Pringle for assistance with preparation of the figures. We also thank Elizabeth Hamel for secretarial assistance and Shirley Buchanan for assistance with the data analysis and for her careful reading of early versions of this manuscript.

Correspondence should be addressed to Donald A. Powell, Neuroscience Laboratory (151A), William Jennings Bryan Dorn Veterans Affairs Medical Center, 6439 Garners Ferry Road, Columbia, SC 29209-1639.

Brian Maxwell's present address: Waccamaw Mental Health Center, P.O. Box 718, Georgetown, SC 29442.

James Penney's present address: Columbia Housing Authority, 1917 Harden Street, Columbia, SC 29204

Copyright (C) 1996 Society for Neuroscience $0270-6474 / 96 / 166296-11 \$ 05.00 / 0$
1994). Similarly, multiple- and single-unit activity in the mPFC are both correlated with acquisition of Pavlovian conditioned HR decelerations using optimum ISIs (Gibbs and Powell, 1988; 1991; Gibbs et al., 1992; Maxwell et al., 1994). No previous studies have assessed mPFC multiple- or single-unit activity using the short ISIs that support EB conditioning. Thus, in the present experiment, multiple-unit activity (MUA) in the mPFC was assessed in rabbits that received classical $\mathrm{EB}$ or NM conditioning.

It has also been previously reported that various nuclei in the amygdala participate in Pavlovian cardiovascular conditioning. Kapp and colleagues (1979) have shown that lesions of the amygdala central nucleus (ACN) greatly attenuate conditioned HR decelerations in the rabbit and that multiple- and single-unit activity in the ACN is associated with the acquisition of conditioned HR responses (Applegate et al., 1982; Pascoe and Kapp, 1985). LeDoux and colleagues have similarly shown that conditioned increases in blood pressure (BP) and HR in the rat are associated with medial geniculate input to the lateral nucleus of the amygdala, which provides second-order neurons to the basolateral and central nucleus (LeDoux, 1994). Damage to this circuit abolishes conditioned HR and BP changes (LeDoux et al., 1986), and neuronal activity in the lateral nucleus is associated with the occurrence of these HR and BP CRs (Clugnet et al., 1990). McCabe and colleagues have shown that this circuit is also intimately involved in conditioned HR decelerations in the rabbit (McCabe et al., 1992), and Davis and colleagues (Davis, 1992) have demonstrated the participation of the ACN and interconnected structures with conditioned enhancement of the startle reflex (i.e., "fear-potentiated startle").

The cerebellar vermis has also long been known to participate 
in cardiovascular, as well as other kinds of autonomic control, as recently reviewed by Ghelarducci and Sebastiani (1996). Moreover, vermal lesions have been shown to impair HR conditioning in both rats (Supple and Leaton, 1990) and rabbits (Sebastiani et al., 1992; Supple and Kapp, 1993). Vermal connections to hypothalamic and brainstem nuclei, e.g., parabrachial nuclei, provide possible anatomical substrates for these effects (Ghelarducci and Sebastiani, 1996), but their functional significance is unknown. Needless to say, the relationship of such cerebellar control of learned autonomic adjustments to similar control by the mPFC and amygdala is also not known at the present time.

Thus, it appears that the mPFC, as well as the amygdala and cerebellar vermis, are intimately involved in the mediation of learned autonomic changes that have been deemed to index emotional changes. However, the common interpretation of prefrontal function as being involved in complicated behavioral processes such as timing and anticipatory and intentional behaviors, etc. (Kolb, 1984; Fuster, 1989; Damasio, 1994), suggests that it could play a more direct role in the integration of autonomic and somatomotor behaviors than either the amygdala or cerebellum. In support of this hypothesis, it has been demonstrated that reversal conditioning of a Pavlovian EB discrimination is greatly impaired by mPFC lesions, whereas lesions of the amygdala have no effect on either acquisition or reversal of this discrimination (Chachich and Powell, 1992, 1994). The strong projections of the mPFC to the neostriatum and the pontine nuclei (Buchanan et al., 1994), which in turn project to the cerebellum [where the deep nuclei (i.e., the interpositus nucleus) have been shown to provide an essential CNS substrate for learned somatomotor behaviors (Thompson, 1991)], provide a possible neuroanatomical substrate for such mPFC-behavioral interactions.

The present study used the rabbit EB and HR classical conditioning models, originally developed by Gormezano (1966) and Schneiderman et al. (1966), to study this problem further by relating $\mathrm{mPFC}$ neuronal activity to both learned somatomotor and autonomic responses. The study was composed of two experiments to determine the extent to which neuronal activity in the $\mathrm{mPFC}$ was associated with acquisition of classically conditioned EB and NM responses. In one experiment, the Pavlovian EB CR was measured using a tone/periorbital shock contingency, and in the other, NM conditioning was assessed using a tone/airpuff contingency. Neuronal activity was assessed in the mPFC during both experiments. A nonassociative pseudoconditioning control group was also used in both experiments, in which the CS and US were presented explicitly unpaired over a similar series of sessions. Because we believe that the mPFC provides an emotional component to learned behavior, we hypothesized that CS-evoked neural activity in the mPFC would be greater in response to the more aversive periorbital shock US than the relatively benign airpuff US.

\section{MATERIALS AND METHODS}

Animals. The animals were 65 experimentally naive New Zealand albino rabbits weighing between 2.2 and $3 \mathrm{~kg}$ on arrival from a local United States Department of Agriculture-approved supplier. The animals were approximately $150 \mathrm{~d}$ old at the beginning of the experiment and were housed in an AAALAC-approved animal facility. Temperature and humidity were continuously controlled, as was a $12 \mathrm{hr}$ light/dark cycle (07:00-19:00 light); all experiments were conducted during the light portion of the cycle. The animals were maintained on ad libitum food and water for the duration of the experiment. During all of this research, the principles for the care and use of laboratory animals, as outlined by the United States Public Health Service, were strictly followed.

Surgery. Electrolytically etched Epoxylite-insulated tungsten microelec- trodes (1-4 M $\Omega$ impedance at $500 \mathrm{~Hz}$ ) were chronically implanted bilaterally in each animal under general anesthesia induced by ketamine hydrochloride $(55 \mathrm{mg} / \mathrm{kg}$, i.m.), supplemented with chlorpromazine hydrochloride $(8 \mathrm{mg} / \mathrm{kg}$, i.m.). The anesthetized animal was positioned in a Kopf stereotaxic instrument, and the dorsal skull was exposed. The position of the skull was adjusted so that bregma was elevated $1.5 \mathrm{~mm}$ with respect to lambda, and the bone overlying the prospective recording site was removed by drilling. Next, the microelectrode tip was advanced under stereotaxic guidance through the exposed dura to the desired location, which varied from animal to animal with respect to the following coordinates: $4.0-8.0 \mathrm{~mm}$ anterior to bregma, $0.4-0.9 \mathrm{~mm}$ lateral to the midline, and 4-6 $\mathrm{mm}$ below dura. Leads from the recording electrode and from a reference screw implanted in the skull over the midsagittal sinus were then connected to an Amphenol socket, which was subsequently secured to the skull with dental acrylic and stainless-steel jeweler's screws. The animal then received an injection of bicillin and was subsequently allowed at least 1 week to recover from the effects of surgery. During this time, it was monitored daily, and antibiotics and/or analgesics were administered as needed to prevent postoperative infection and discomfort.

Histological procedures. After training was complete, the recording site was marked by passing an anodal current of $20 \mu \mathrm{A}$ through the recording electrode for $20 \mathrm{sec}$. The animal was then killed with sodium pentobarbital and perfused intracardially with physiological saline followed by $10 \%$ formalin. After immersion fixation in a cryoprotectant solution (Rosene et al., 1986), the brain was blocked in the transverse plane, and frozen serial sections were taken at $40 \mu \mathrm{m}$ through the prefrontal region. The sections were then counterstained with thionin. After air drying and coverslipping, the electrode tract and electrolytic lesion were located on line drawings made of the appropriate sections using a Leitz drawing tube.

Apparatus and recording procedures. The apparatus and procedures for classical conditioning in our laboratory have been described previously (Powell and Levine-Bryce, 1988). Briefly, the animals were tested in sound-deadening and ventilated commercial chambers in standard Plexiglas rabbit restrainers (Gormezano, 1966). Experimental contingencies were controlled by a PDP-11/23 microcomputer connected to a Grass model 7 polygraph, which was used to record the HR and EB responses. For one set of animals, the conditioned stimulus was a $1.25 \mathrm{sec}$ tone of 75 $\mathrm{dB}$ (sound pressure level). The US was an alternating current electric shock train of $\sim 3 \mathrm{~mA}$ intensity and $250 \mathrm{msec}$ (eyeshock group). A $1.1 \mathrm{sec}$ tone was the CS for a second group, and a 3 psi airpuff of $100 \mathrm{msec}$ duration was the US (airpuff group). The ISI in all cases was thus $1.0 \mathrm{sec}$.

The HR and EB responses were recorded as described previously (Powell and Joseph, 1974; Powell and Levine-Bryce, 1988). Stainless steel safety pins inserted subcutaneously over the right front leg and left flank served as electrocardiographic electrodes. Carefully matched electrodes constructed of stainless steel orthodontic wire were inserted between the upper and lower eyelids for simultaneously recording eyelid closure and NM extension (i.e., the corneoretinal potential or EB response) in the eyeshock group. EB sensitivity was set to give a $1 \mathrm{~mm}$ pen deflection per $100 \mu \mathrm{V}$ potential across the recording electrodes. This signal was integrated over the CS interval by a Grass Model 7P3 AC preamplifier and integrator. EB CRs were defined as those responses of $100 \mu \mathrm{V}$ or greater ( $1 \mathrm{~mm}$ polygraph pen deflection) that occurred during the CS-US interval. This results in an eyelid movement of $\sim 0.50 \mathrm{~mm}$. The NM response was measured in the airpuff group using a tranducer developed by Gormezano and Gibbs (1988). It consists of a ball-and-socket arrangement that is directly coupled to the NM through a fine silk suture. The latter is attached by means of a rigid wire to a piece of polarized film, through which a beam of light travels in a light-tight plastic tube. Displacement of the film by the directly coupled ball-and-socket arrangement changes the amount of light that passes through a second, fixed piece of polarized film, also contained in the light-tight tube. This change in illumination results in a voltage change produced by a photocell, which can then be detected and directly related to the magnitude of the NM response. The NM CR was also defined as a response occurring during the CS of $\geq 0.5 \mathrm{~mm}$. EB and NM CR latency were defined as the time period between CS onset and the occurrence of a criterion voltage change. Response amplitude was the highest voltage recorded during the CS period. MUA was recorded as described in previous papers (Gibbs and Powell, 1988). MUA was conventionally amplified (World Precision Instruments Model Dam-50), bandpass-filtered (0.5-10 kHz), and fed to an amplitude-window discriminator (World Precision Instruments Model 121) for signal analysis. Lists of times of occurrence of discriminable 
neuronal discharges were generated by the on-line computer, and peristimulus time histograms were constructed around the pre-tone and tone periods, as described below. The window discriminator was set for each animal so that the pre-tone baseline activity recorded was $\sim 50 \mathrm{~Hz}$. MUA and HR activity were displayed on an oscilloscope and acoustically monitored to ensure that any subtle movement artifacts or baseline shifts could be documented and thus excluded from the data analysis.

Conditioning procedures. The airpuff and eyeshock groups were each divided into two subgroups. One subgroup received systematic pairings of tone and US and will hereafter be referred to as the "conditioning" groups. A second subgroup, which served as a "pseudoconditioning" or "nonassociative" control group, received the same number of tones and shocks (or airpuffs) as the conditioning groups; however, in this case, the tones and USs were presented in an unpaired, pseudorandom sequence according to a predetermined schedule. The intertrial interval for the latter groups was half that of the conditioning groups. Total session length was thus identical for the two groups in all cases. EB and NM responses were measured during each tone as described above. HR was also measured on each trial by starting the real-time clock in the computer with the first QRS complex that occurred at the beginning of a $4 \mathrm{sec}$ period preceding the tone. Interbeat intervals (IBIs) were measured by the clock until tone termination. On the day before the initiation of conditioning, each animal received 10 tone-alone presentations to allow the cardiac component of the orienting reflex to habituate. After the orienting and habituation session, each animal received four daily sessions of conditioning (60 trials) or pseudoconditioning (120 trials), followed by two sessions of extinction training during which tones-alone were again presented (60 trials each). The intertrial interval was $60( \pm 30)$ sec for the conditioning groups and $30( \pm 15) \mathrm{sec}$ for the nonassociative control groups.

Data reduction and statistical analysis. IBI duration was assessed as described above for 10 IBIs before tone onset and for up to three IBIs after tone onset. Each IBI was converted to $\mathrm{HR}$ in beats per minute (BPM). The pre-tone HR for each IBI was averaged to yield a single baseline HR value. The HR CR was then obtained by subtracting this mean from the HR associated with each post-tone IBI. MUA was evaluated by compiling peristimulus time histograms for selected blocks of trials (see below) in bins of $20 \mathrm{msec}$ over the $1 \mathrm{sec}$ period immediately before (baseline period) and after (tone period) tone onset. $Z$-score equivalents for each of the tone bins were calculated by subtracting the mean discharge of a specified bin during the baseline period associated with a block of trials from the discharge evoked by the tone associated with the corresponding bin, and dividing this difference by the SD of the baseline discharge, as described in previous reports (Gibbs and Powell, 1988). The HR, EB or NM, and $Z$-score data were submitted to separate mixed-design repeated measures ANOVAs, generally involving the nonrepeated factor of group (paired vs unpaired CS/US), and repeated measures of interval during the tone (in IBIs or msec) and trial (or trial-block). Additional post hoc and correlational analyses are detailed below.

\section{RESULTS}

\section{Histology}

Area 32 (prelimbic area) of the mPFC has been shown to be necessary for HR conditioning in the rabbit (Powell et al., 1994). Consequently, the electrode tips were aimed for this region of the mPFC. However, histological analysis of the animals used in the two experiments revealed that some electrode tips were located in areas 8,24 , or 25 , which are the most dorsal and ventral regions of the mPFC, respectively (Buchanan et al., 1994). Because these were few in number $(4,5$, and 2 , respectively), the data from these sites were discarded, and the behavior of these animals is not reported. The electrode tips for the animals included in the study are shown in Figure 1. The top panel shows the recording sites of animals that received eyeshock conditioning, and the bottom panel shows those that received airpuff conditioning. Electrode placements of animals in the conditioning groups are indicated by circles and the pseudoconditioning groups by Xs. This figure shows that the recording sites were located primarily in area 32 , with a few on the border of areas 32 and 24. Electrode tips were located primarily in the middle and deep layers of the cortical tissue, although several were also located in the superficial layers. A preliminary analysis of the evoked MUA of the different layers revealed no difference between the middle and deep layers. There were too few placements in the superficial layers (primarily layer 1) for a statistical analysis, and these animals were thus also excluded ( $n=6$ in the conditioning groups and $n=4$ in the pseudoconditioning groups). This resulted in 23 and 18 placements in the paired and unpaired eyeshock groups and 21 and 17 placements in the paired and unpaired airpuff groups, respectively.

\section{Unconditioned HR and EB responses}

Tone-evoked changes in the HR, EB, and NM responses during pretraining did not differ between the conditioning and pseudoconditioning groups. As has been obtained in previous experiments (Powell and Levine-Bryce, 1988), few EB responses were obtained in any animals. As has also been found in many previous experiments (Powell and Levine-Bryce, 1988), the HR response initially consisted of a relatively large HR deceleration, especially during the first trial. This response, referred to as the cardiac orienting reflex, reached its greatest magnitude during the fifth or sixth IBI on the first trial in both groups of animals. The magnitude of the orienting reflex declined predictably across repeated tone-alone presentations and appeared to be fully habituated by the end of pretraining for all animals. The results of ANOVAs applied to these data confirmed that the HR change over trials was significant for both the eyeshock $\left(F_{(9,256)}=4.60\right.$; $p<0.001)$ and airpuff $\left(F_{(9,208)}=2.91 ; p<0.003\right)$ groups. However, there were no significant differences between the groups that would later receive paired and unpaired training for either the eyeshock or airpuff conditions.

\section{Tone-evoked mPFC neuronal changes}

As also obtained in previous studies (Gibbs and Powell, 1988), initial presentations of the tone alone during orienting reflex assessment elicited increases in MUA in animals later assigned to both the NM and EB conditioning groups. However, these CSevoked changes in MUA habituated over trials, as also reported in several previous papers (Powell et al., 1990; Gibbs et al., 1992; Maxwell et al., 1994). ANOVA of $Z$-scores averaged over trials 1-3, 4-6, and 7-10 yielded significant bin and trial-block effects (all $p$ values $<0.01$ ), but no group effects or group interactions (all $p$ values $>0.10$ ) for either the NM or EB analyses.

\section{EB conditioning and extinction}

Percent EB (top) and NM (bottom) CRs elicited during the tone is illustrated in Figure 2, as a function of the four conditioning sessions and the two extinction sessions. This figure indicates that a systematic increase in both NM and EB CRs occurred as a function of sessions in both the eyeshock and airpuff conditioning groups; however, little change occurred in the nonassociative control groups, although the overall level of responding in these control groups is somewhat higher than that reported previously (Gormezano, 1966), probably because of the longer ISI used in the present study. ANOVA of these data revealed significant group and group $\times$ session effects for both the eyeshock and airpuff analyses (all $p$ values $<0.001$ ). As also indicated in Figure 2 , percent responses declined considerably over the two extinction sessions, although they did not completely extinguish to pretraining levels. ANOVA again revealed significant differences between the conditioning and pseudoconditioning groups for both the airpuff and eyeshock conditions (all $p$ values $<0.01$ ).

Analysis of EB and NM CR latencies and response magnitudes also revealed significant differences between the paired and un- 

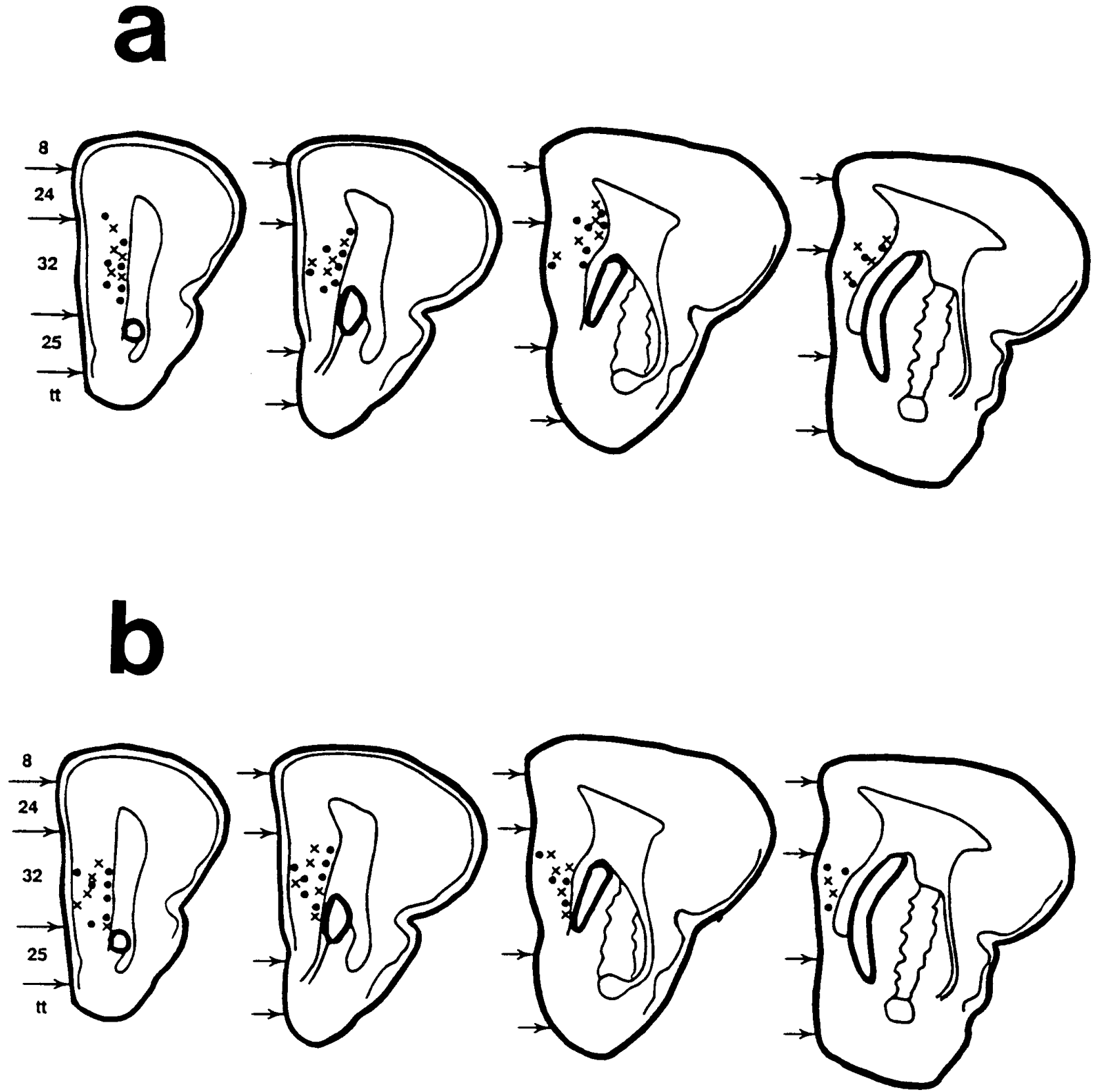

Figure 1. Location of electrode tips for multiple-unit recording electrodes in the mPFC of rabbits that received periorbital shock $(a)$ or airpuff ( $b$ ) as unconditioned stimuli during Pavlovian EB and NM conditioning. Filled circles represent sites of animals that received paired CS/US training, and Xs represent sites of animals that comprised a nonassociative control group that received the same number of CS and US presentations, but they were explicitly never paired with each other. Brodmann areas: 8 , frontal eye fields; 24 , anterior cingulate cortex; 32 , prelimbic area; 25 , infralimbic area. $t t$, Tenia tecta.

paired groups. These data are shown in Table 1. The top two panels show mean CR latencies and amplitudes as a function of acquisition and extinction sessions for the paired and unpaired groups that received eyeshock as the US, and the bottom two panels show similar data for the groups that received airpuffs as the US. As can be seen in Table 1, both latency and amplitude increased over sessions for the paired, but not the unpaired, groups during acquisition, but not during extinction training. ANOVA of these data revealed significant group $\times$ session interactions for both the eyeshock and airpuff groups during acquisition, indicating that these differences were reliable. These differences were also significant for extinction, but only for the amplitude data (all $p$ values $<0.05$ ). Some differences between the two types of responses are also notable. For example, the early latencies for the NM response are considerably shorter than those of the EB response. In general, CR amplitude was also smaller for the NM response. However, these differences were probably attributable to the different measurement techniques used (see above) and not to any intrinsic differences between the two types of responses.

\section{Associative and nonassociative effects on HR conditioning}

Figure 3 shows mean HR change as a function of three consecutive IBIs during the CS for each of the four sessions of acquisition 

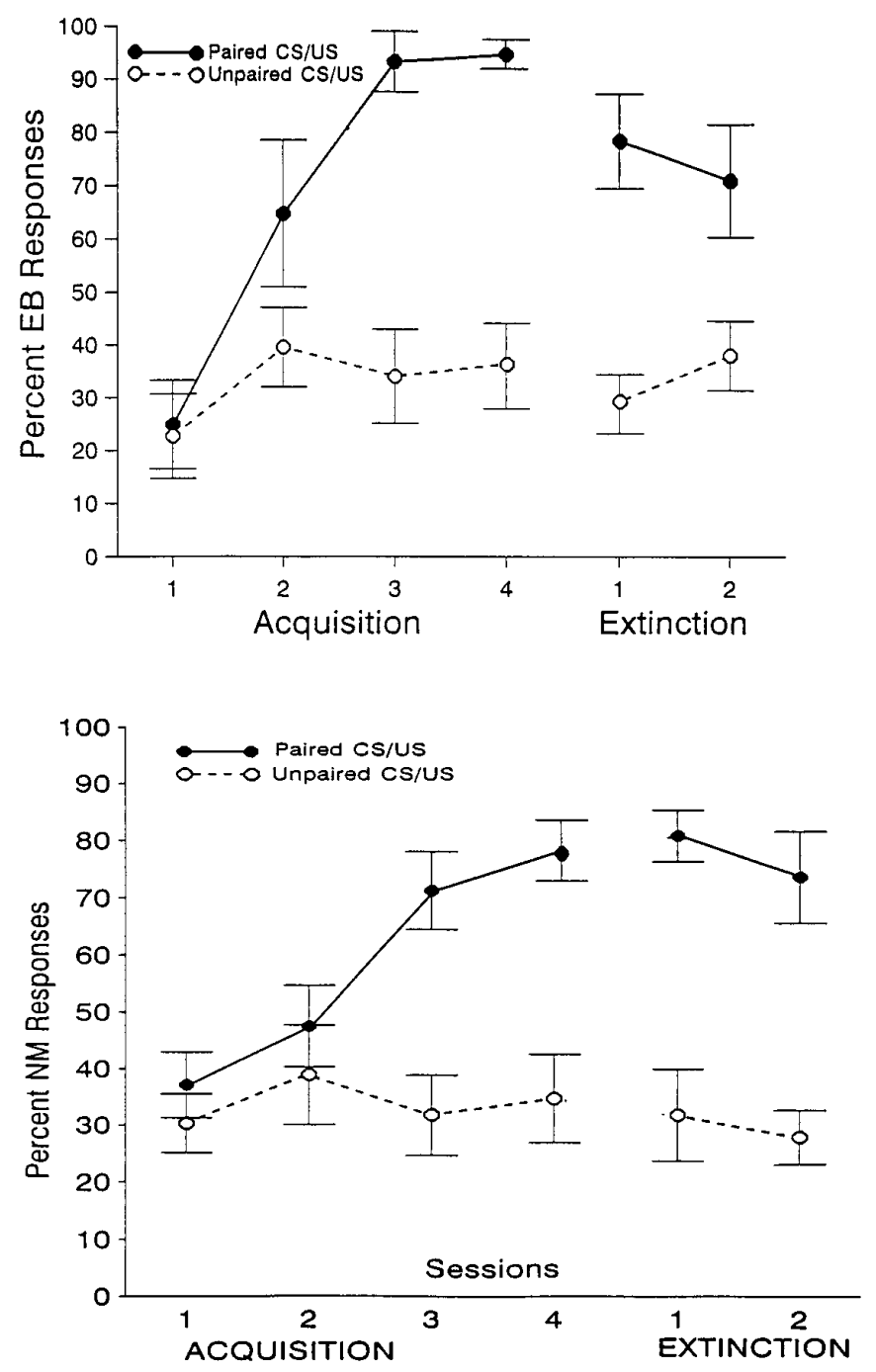

Figure 2. Percent change in Pavlovian conditioned EB (top) and NM (bottom) CRs as a function of four sessions of acquisition and two sessions of extinction training. Periorbital shock was the US for EB conditioning, and an airpuff was the US for NM conditioning. In each case, one group of animals received paired CS/US training, and a second, nonassociative control group received explicitly unpaired presentations of the CS and US.

training for the eyeshock (top) and airpuff (bottom) groups. Toneelicited change in HR during acquisition training consisted of a decrease in HR of 5-10 BPM in the paired eyeshock group, which increased in magnitude over sessions, except for the last session, when it became somewhat smaller. Tone-evoked HR changes in the explicitly unpaired group, however, consisted of accelerative responses throughout training. The decrease in HR in the paired group was apparent even during the first session of training and became considerably greater in the conditioning compared with the pseudoconditioning group by the end of the first session. ANOVA of these data revealed significant group $\left(F_{(1,12)}=6.02\right.$; $p<0.03)$, as well as session $\left(F_{(3,32)}=8.4 ; p<0.003\right)$ and group $\times$ IBI $\left(F_{(6,64)}=4.28 ; p<0.001\right)$ effects. As shown in the bottom panel of Figure 3, however, the change in HR in the airpuff group was considerably smaller and, as has been found in previous experiments (Powell et al., 1993), the differences between the HR response of the conditioning and pseudoconditioning airpuff groups were not significantly different $\left(F_{(1,26)}=1.3 ; p<0.3\right)$, although the IBI effect was significant $\left(F_{(2,52)}=10.9 ; p<0.001\right)$. ANOVA of the extinction data (not shown in Fig. 4) also revealed significant group differences for the eyeshock $\left(F_{(1,12)}=6.8 ; p<\right.$ $0.001)$, but not the airpuff groups $(p>0.10)$.

\section{Associative and nonassociative effects on MUA}

Figure 4 shows tone-evoked changes in MUA in the mPFC of the eyeshock groups (top) and airpuff groups (bottom). These data are shown as mean $Z$-score equivalents, as described above, for 20 msec bins computed over blocks of 20 trials each (i.e., "trial blocks"). The data are plotted as a function of the first fifteen 20 msec bins (of the $1 \mathrm{sec}$ measurement interval) averaged over three trial blocks of 20 trials each for each of the four sessions of the experiment. As can be seen in Figure 4, tone/eyeshock pairings served to enhance the tone-evoked responses recorded at $\mathrm{mPFC}$ placements. This response consisted of an initial brief increase in activity, followed by a brief inhibitory component. Neither of these early neuronal changes appeared to be associative, however, because neither changed appreciably over sessions and neither appeared to be different for the paired and unpaired groups. However, a longer-duration gradual increase in discharge occurred later, which became larger over sessions and was considerably greater in the paired than unpaired groups, thus suggesting that this later component was associatively produced. This component was much greater toward the end of training, when, as indicated in Figure 2, EB conditioning had reached its maximum levels. Although tone-evoked activity in the pseudoconditioning group showed the initial inhibitory component, it did not show the later increases in neuronal activity exhibited by the paired group. An ANOVA computed on these data supports these observations. The dimensions in this analysis included group (2 levels), bin (15 levels), and the trial blocks over which the $Z$-scores were calculated (12 levels, i.e., 3 blocks per session). ANOVA of the eyeshock data revealed significant group $\left(F_{(1,36)}=5.79 ; p<0.02\right)$, bin $\left(F_{(14,448)}=5.42 ; p<0.0001\right)$, block $\left(F_{(11,369)}=3.29 ; p<\right.$ $0.0003)$, group $\times$ bin $\left(F_{(14,448)}=3.0 ; p<0.001\right)$, and group $\times$ block $\left(F_{(11,369)}=2.28 ; p<0.01\right)$ effects. In general, tone-evoked MUA appeared to be greater in the airpuff groups compared with the eyeshock groups, especially for the unpaired group during later sessions. However, as Figure 4 (bottom) shows, the differences between the conditioning and pseudoconditioning airpuff groups appears to be negligible. Although an initial ANOVA revealed a significant group $\times$ block $\times$ bin interaction in the airpuff group $\left(F_{(154,5382)}=1.28 ; p<0.01\right)$, after application of the Greenhouse-Geisser (1959) correction for sphericity, this interaction was no longer significant $(p>0.10)$. These findings thus show that, although both the airpuff and eyeshock groups revealed significant increases in EB and NM conditioning over sessions, increases in $\mathrm{mPFC}$ neuronal activity, as a result of $\mathrm{CS} / \mathrm{US}$ pairings, were elicited only in the eyeshock group.

\section{MUA activity during extinction}

CS-evoked activity showed a decline in the eyeshock group during extinction (not shown). Again, significant group $\left(F_{(1,32)}=15.1\right.$; $p<0.0001)$, bin $\left(F_{(14,448)}=10.0 ; p<0.001\right)$ and group $\times$ bin $\left(F_{(14,448)}=2.16 ; p<0.01\right)$ effects occurred. Post-tests comparing MUA in the paired and unpaired groups during the second session of extinction revealed no significant group effects, indicating that CS-evoked MUA effects in the conditioning group had declined to that of the pseudoconditioning group during extinction. 
Table 1. Mean ( \pm SEM) latencies $(\mathrm{msec})$ and amplitudes $(\mathrm{mV})$ of eyeblink and NM-conditioned responses of rabbits that received either paired or unpaired CS/US conditioning training

\begin{tabular}{|c|c|c|c|c|c|c|}
\hline & \multicolumn{4}{|c|}{ Acquisition sessions } & \multicolumn{2}{|c|}{ Extinction sessions } \\
\hline & \multicolumn{6}{|c|}{ EB (eyeshock US) } \\
\hline & 1 & 2 & 3 & 4 & 1 & 2 \\
\hline Paired & $423.8(5.4)$ & $448.8(4.7)$ & $436.0(2.9)$ & $447.3(3.1)$ & $434.0(4.6)$ & $426.0(3.7)$ \\
\hline Unpaired & $428.0(5.8)$ & $423.0(3.9)$ & $429.0(3.8)$ & $428.0(5.8)$ & $434.0(7.8)$ & $427.0(5.2)$ \\
\hline \multicolumn{7}{|c|}{ (B) Amplitude } \\
\hline
\end{tabular}

NM (airpuff US)

\begin{tabular}{|c|c|c|c|c|c|c|}
\hline \multicolumn{7}{|l|}{ (A) Latencies } \\
\hline Paired & $137.0(27.6)$ & 291.8 (34.2) & $412.2(16.2)$ & 466.0 (12.4) & $327.0(29.6)$ & $286.0(33.2)$ \\
\hline Unpaired & $191.0(29.0)$ & $165.0(21.0)$ & $166.0(20.8)$ & $160.0(20.8)$ & $181.0(24.0)$ & $219.0(26.7)$ \\
\hline \multicolumn{7}{|c|}{ (B) Amplitude } \\
\hline Paired & $0.17(0.06)$ & $0.62(0.20)$ & $2.23(0.55)$ & $1.86(0.26)$ & $0.85(0.16)$ & $0.86(0.17)$ \\
\hline Unpaired & $0.36(0.15)$ & $0.51(0.17)$ & $0.47(0.12)$ & $0.57(0.15)$ & $0.35(0.12)$ & $0.54(0.22)$ \\
\hline
\end{tabular}

Periorbital electric shock (eyeshock) was the US for EB conditioning, and a corneal airpuff was the US for NM conditioning.

\section{Relationships between MPFC neuronal activity and conditioned HR and EB responses}

Pearson Product Moment Correlation Coefficients were used to determine whether CS-evoked MUA in the mPFC was related to the behavioral responses assessed during conditioning. Separate coefficients were computed for each session using the mean $Z$-scores associated with the last (15th) bin for each session as the neuronal activity index and the mean HR change from baseline associated with the third IBI during the CS as the index of HR conditioning. These analyses yielded significant negative correlations for sessions 3 and 4 ( $r s=-0.64$ and -0.53 , df $=17$, all $p$ values $<0.05$, respectively) for the paired shock group, indicating that larger increases in MUA were associated with greater decelerative HR changes in this group. No correlations were significant, however, in the unpaired shock group or the airpuff groups. Similar correlations with the percent EB scores were also not significant in any group. Moreover, neither the HR nor the EB scores were correlated with MUA during pretraining or extinction in any group. Thus, CS-evoked MUA and HR changes were correlated, but only during the conditioning phase of training, and only then in the group that showed associative HR CRs, i.e., the group that received periorbital shock as the US.

\section{Baseline HR and MUA}

Although the window discriminator for detecting neuronal spikes was set at the beginning of each session so that average pre-tone baseline MUA for the $1 \mathrm{sec}$ period preceding the tone was $\sim 50$ $\mathrm{Hz}$, as has been observed in previous experiments (Maxwell et al., 1994), there was a tendency for baseline MUA to increase during the session. However, ANOVA revealed that this effect did not reach statistical significance in any group, nor did any group interact with this variable ( $p>0.10$ in all comparisons). ANOVA of mean pre-CS baseline HR scores also revealed no significant group effects among either the shock or airpuff groups. Mean HR $( \pm \mathrm{SE})$ was $218( \pm 17), 236( \pm 21), 212( \pm 12)$, and $224( \pm 15)$ BPM for the shock-paired, shock-unpaired, airpuff-paired, and airpuffunpaired groups, respectively.

\section{DISCUSSION}

Our original hypothesis that CS-evoked mPFC neuronal activity would be augmented by classical conditioning, in which eyeshocks, but not airpuffs, served as USs was confirmed, because MUA evoked by the CS increased significantly only when the more aversive eyeshock was used as the US. Although CS-evoked changes in MUA occurred in the groups that received the airpuff as the US, these increases were considerably smaller than those in the animals that received eyeshock as the US and were not significantly different from that observed in animals that received explicitly unpaired presentations of the CS and US. In this regard, it is important to note that whereas conditioned HR decelerations were also observed in the eyeshock group, changes in conditioned $\mathrm{HR}$ in the airpuff group (although decelerative in character) were again not significantly different from those obtained in the explicitly unpaired nonassociative control group. The decelerative HR CR obtained in the eyeshock groups, on the other hand, appears to be typical of that obtained in previous experiments (Powell and Kazis, 1976). Thus, it increased in magnitude as a function of early training, but was somewhat smaller during the last session when CS-evoked EB responding was maximal, presumably because of increased somatomotor demands on the cardiovascular system produced by EB CR occurrence. As also obtained in previous studies (Gibbs and Powell, 1988), the tone-evoked HR response in the unpaired animals receiving eyeshock consisted of mild tachycardia.

It has been shown in several previous experiments that CSevoked increases in MUA occur in areas 8, 24, and 32 of the mPFC in rabbits while the animals are undergoing classical HR conditioning (Gibbs and Powell, 1988; Gibbs et al., 1992; Maxwell et al., 1994). In these experiments, however, the ISI (i.e., the interval between the onset of CS and US) was considerably longer than that used in the present experiments. In the present study, a $1 \mathrm{sec}$ ISI was used, which, although not optimal for either EB or HR conditioning, nevertheless yields robust EB and NM conditioning, as revealed by the present results. Moreover, a $1 \mathrm{sec}$ ISI also allows sufficient time to assess the HR CR over at least three post-CS IBIs for most animals. As noted above, this response in 

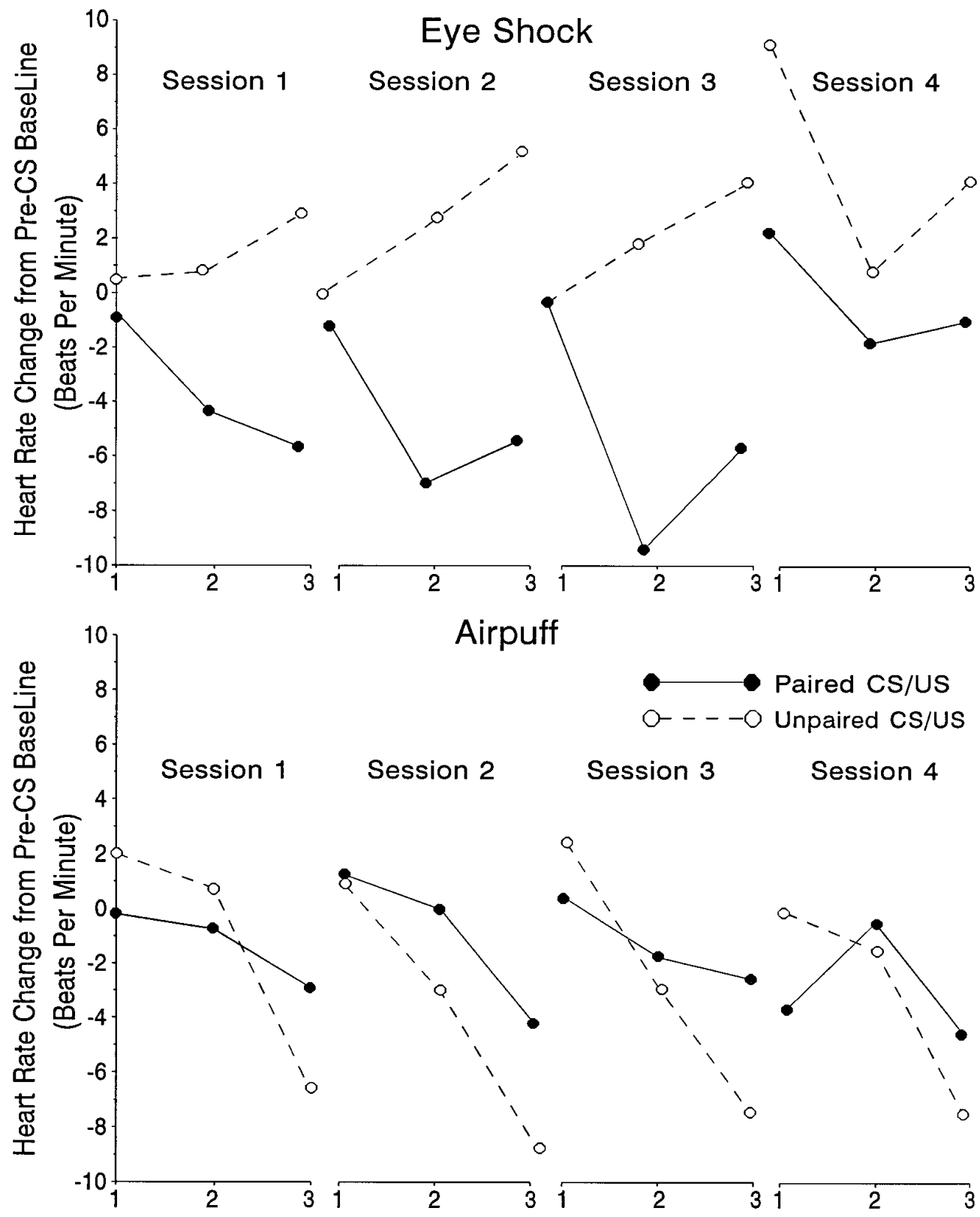

Airpuff

Figure 3. Mean change in HR (BPM) from pre-CS baseline that occurred during the $1 \mathrm{sec}$ tone presentation for animals that either received eyeshock (top) or a corneal airpuff (bottom) as the US during Pavlovian EB or NM conditioning, respectively. Data are shown for three post-CS IBIs over each of four acquisition sessions for paired CS/US and explicitly unpaired CS/US groups in each case.

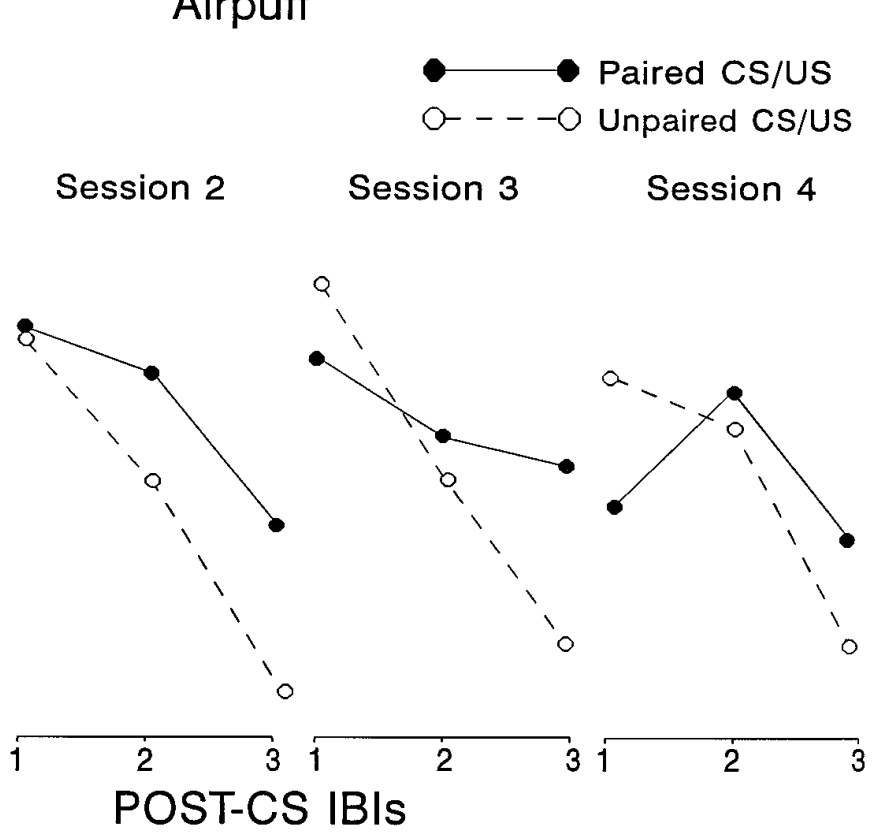

the restrained rabbit has invariably been shown to be cardiac slowing (Powell and Kazis, 1976; Powell and Levine-Bryce, 1988). An important finding in the present study is that, whereas CSevoked increases in $\mathrm{mPFC}$ neuronal activity were obtained in the animals that received eyeshock as the US, no associative changes in neuronal activity were observed in the less aversive airpuff group. Nevertheless, both groups of animals revealed normal EB and/or NM conditioning. Thus, it is apparent that mPFC MUA is not related specifically to classically conditioned skeletal CRs, but instead appears to be associated with the occurrence of an aversive CS/US contingency, i.e., in the present instance the toneeyeshock pairings. However, airpuff URs, if of sufficient magnitude, have been demonstrated to elicit decelerative HR changes comparable to that of eyeshock URs (McEchron et al., 1991). mPFC MUA elicited by tone CSs, paired with such high-intensity airpuffs, thus might also be expected to show increases during training, because it is probably the aversiveness associated with the US, and not the type of US used per se, that is critical for mPFC processing. In any case, it is apparent that CS-evoked increases in neuronal activity in the $\mathrm{mPFC}$ do not distinguish between skeletal conditioning elicited by different kinds of USs at this relatively long ISI, but appear to be specifically associated with the more aversive CSs paired with eyeshock as the US.

These findings also suggest that although the mPFC may be important for HR conditioning, it is not necessary for concomitantly occurring skeletal conditioning, at least when simple delay conditioning is involved. As discussed in the introductory remarks, a host of other data supports this conclusion. For example, lesions of the mPFC in the rat abolish conditioned HR increases and pressor responses in the free-moving animal (Frysztak and Neafsey, 1994), and we have shown in several experiments that damage to the mPFC also produces greatly attenuated conditioned bra- 


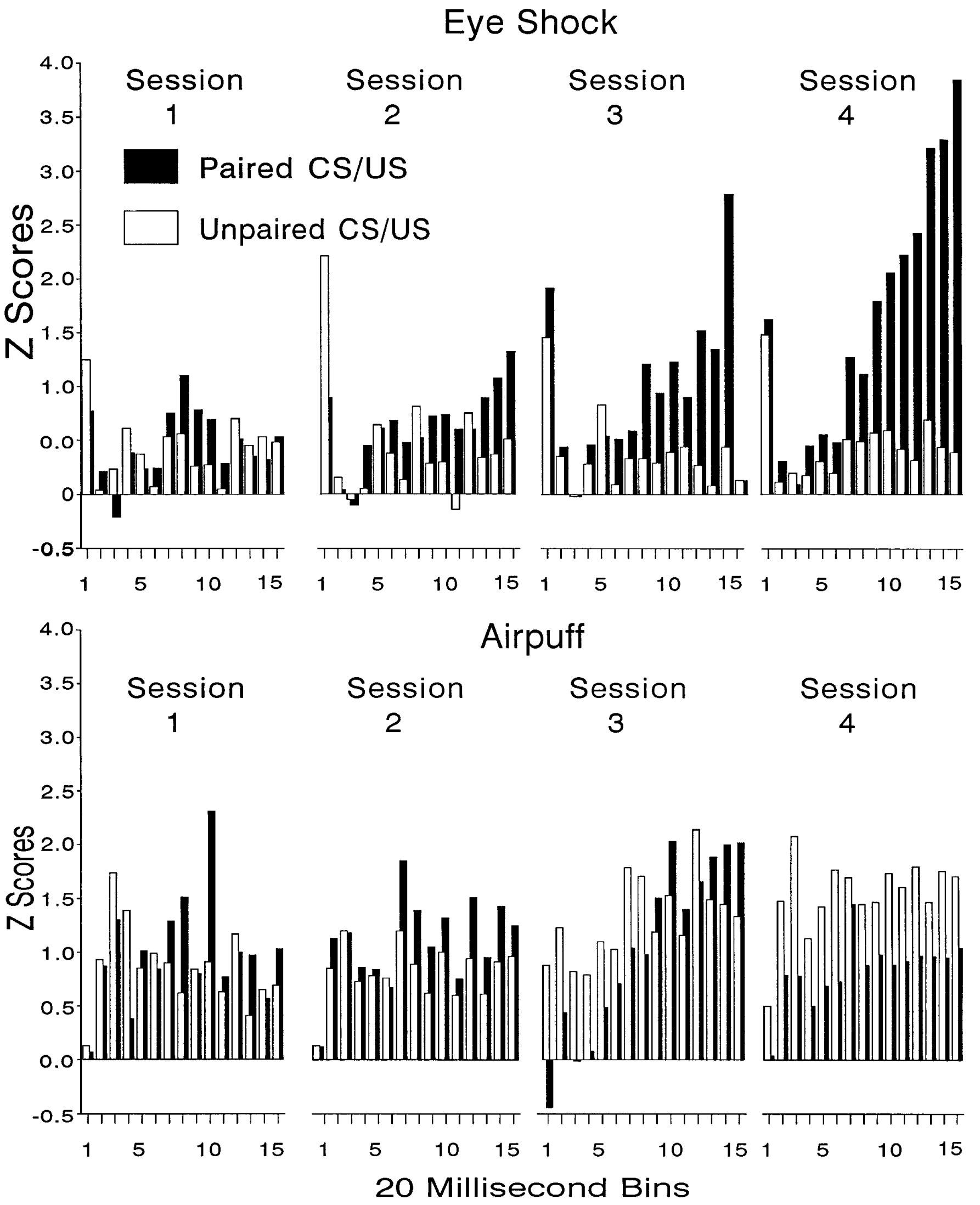

Figure 4. Tone-evoked changes in MUA in the mPFC of rabbits that received Pavlovian EB conditioning, in which periorbital electric shock served as the US, or that received Pavlovian NM conditioning, in which a corneal airpuff served as the US. Data are shown for a paired CS/US group and for an explicitly unpaired CS/US group as a function of $20 \mathrm{msec}$ bins over four acquisition sessions of 60 trials each. The data are shown as $Z$-score equivalents that were normalized for pre-CS baseline activity. 
dycardia in the restrained rabbit; in many animals, this learned response is completely abolished (Buchanan and Powell, 1982; Powell et al., 1994). Previous lesion experiments, although few, also suggest a lack of involvement of the mPFC in skeletal conditioning (Buchanan and Powell, 1982). However, there are data that suggest the participation of the mPFC in skeletal conditioning under conditions that are not optimal for acquisition of the response. A recent preliminary report (Buchanan et al., 1994), for example, suggests that damage to the mediodorsal nucleus of the thalamus (MDN), which is the thalamic projection nucleus to the prefrontal region, has a profound effect on EB conditioning under conditions that are not optimal for acquisition. For example, when relatively long ISIs were used, differences between lesioned and sham animals on EB conditioning were greatly exaggerated. Manipulation of the schedule of reinforcement also severely impaired EB conditioning in animals with MDN lesions. It has been known for some time that classical EB conditioning is greatly affected by lean schedules of reinforcement (Powell and Milligan, 1975), and Buchanan et al. (1994) reported a severe attenuation of acquisition in MDN-lesioned animals when the reinforcement schedule allowed for reinforcement on only $25 \%$ of the trials. Damage to MDN also impairs differential conditioning of the HR response as well as reversal conditioning of an originally learned discrimination using the EB CR as the response criterion (Buchanan, 1991).

We have also recently shown that lesions of the mPFC produce a similar effect (Chachich and Powell, 1992). In this study, alluded to in the introductory remarks, it was found that mPFC damage to areas 24 and 32 had minimal effects on original EB discrimination during differential conditioning, but greatly increased the time required for rabbits to reach a reversal criterion of a $30 \%$ difference in responding to a new $\mathrm{CS}+$ and $\mathrm{CS}-$. In fact, only one of eight mPFC-lesioned animals met this criterion, whereas eight of eight animals with sham lesions did so. Thus, it is clear from these and similar findings that the mPFC may indeed have an influence on somatomotor response selection, although under optimal conditions little effect is noted (Buchanan and Powell, 1982). These findings are compatible with the conclusions by others (Fuster, 1989; Goldman-Rakic, 1990; Damasio, 1994; Devinsky et al., 1995) that the prefrontal cortex is involved not only in stimulus processing, as would be expected based on its role in classical conditioning, but also in appropriate skeletal-response selection. Thus, it would be interesting to determine the extent to which CS-evoked changes in neuronal activity in the mPFC would occur under conditions in which the parameters are less than optimal for acquisition of the EB CR.

Although the present data implicate the mPFC in processing an emotional component of learning, they do not speak to possible differences between mPFC processing of this kind of information and similar processing by other brain structures. As noted above, these include both the amygdala (LeDoux, 1994) and cerebellar vermis (Supple and Kapp, 1993). The efferent connections of the amygdala and mPFC are almost identical (cf. Schwaber et al., 1982; Neafsey et al., 1986; Buchanan et al., 1994). Both provide efferents to the lateral hypothalamus, midbrain central grey, and the autonomic regulatory nuclei in the medulla, all of which are CNS sites associated with elicitation of autonomic changes. As noted, the vermis has similar connections with some of these same structures (Ghelarducci and Sebastiani, 1996). Other studies on a variety of species have also implicated the amygdala and $\mathrm{mPFC}$ in learned responses with obvious emotional components (Bachevalier and Mishkin, 1986; Gaffan and Murry, 1990). As indicated, much evidence suggests that $\mathrm{mPFC}$ processing of emotional stim- uli may have an impact on a somatomotor response selection process that is based on a wide array of other kinds of information, also being processed by the mPFC. However, because this latter information is not available to the amygdala or cerebellum, these structures are presumably not directly involved in such decision making and function more as subcortical substrates for relatively "pure" emotional learning.

There is a great deal of evidence showing that amygdala damage permanently prevents expression of the autonomic and somatomotor responses usually used to index emotional changes (Kapp et al., 1991; LeDoux, 1994). However, other findings suggest preserved retention of behaviors presumably dependent on processing of emotional stimuli by the amygdala, such as inhibitory avoidance training, even after large amygdala lesions including both the basolateral and central nuclei (Parent et al., 1995). The role of the mPFC in the expression of such motivated behaviors is unknown at the present time. However, simple delay EB conditioning, even using the nonoptimal 1 sec ISI used here, apparently does not require $\mathrm{mPFC}$ input for normal acquisition of the response. An EB or NM paradigm requiring limbic or cortical involvement, such as trace or differential conditioning (Buchanan and Powell, 1980; Moyer et al., 1990), would thus appear to be necessary to demonstrate differential amygdala/mPFC participation in acquisition of learned somatomotor behaviors using the present animal model. The amygdala and mPFC of course are also interconnected, primarily via the basolateral nucleus of the amygdala, (Porrino et al., 1981; McDonald, 1987; Barbas and de Olmos, 1990), suggesting that the amygdala and mPFC may jointly participate in the elicitation of autonomic changes that support ongoing skeletal behaviors.

The present data thus support the hypothesis that mPFC cells mediate a learned emotional component to behavior, assuming that the CS-evoked HR changes affected by mPFC manipulations reflect processing of emotional stimulation. A wide array of clinical data also support this hypothesis. For example, Damasio et al. (1990) report data suggesting that the normal affective valence required for guiding complex human behavior is missing in patients with ventromedial prefrontal damage. Appropriate autonomic behaviors are also altered in these patients (Fuster, 1989; Damasio, 1994). The widely reported clinical finding that frontal lobotomy and cingulectomy greatly affects emotional responding also supports this idea (Powell, 1979). The finding that the latter procedure eliminates the emotional, but not the sensory, component of the pain response seems especially important in this regard (Powell, 1979). The specific role that autonomic adjustments might play in these kinds of complicated behaviors is at the present time unclear. Although it is possible that learned autonomic changes are simply indicative of an affective component of learning and memory, as some have suggested (Kapp et al., 1979; LeDoux et al., 1986), it is also possible that they play a causal role in appropriate response selection and thus have adaptive significance in their own right (Lacey and Lacey, 1974; Albiniak and Powell, 1980; Joseph and Powell, 1980). The fact that cardiac inhibition is associated with attentional phenomena (Lacey and Lacey, 1974) and that the mPFC obviously participates in attentional processes (Posner et al., 1988) suggest that the latter hypothesis has merit. However, more data will be required to answer this question definitively.

In any case, it is apparent from the present data that both mPFC neuronal activity and HR changes are relatively unaffected by parametric conditions that promote rapid skeletal conditioning, but for which there is little affective impact of conditioning, 
i.e., when a mild airpuff is the US. On the other hand, when the affective impact is considerably greater, i.e., when eyeshock is the US, considerable increases in mPFC neuronal activity, and large bradycardiac CRs, occur. These data are compatible with the characterization of the mPFC as a cortical site for integration of learned emotional changes.

\section{REFERENCES}

Albiniak BA, Powell DA (1980) Peripheral autonomic mechanisms and Pavlovian conditioning in the rabbit (Oryctolagus cuniculus). J Comp Physiol Psychol 94:1101-1113.

Applegate CD, Frysinger RC, Kapp BS, Gallagher M (1982) Multiple unit activity recorded from amygdala central nucleus during Pavlovian heart rate conditioning in rabbit. Brain Res 238:457-462.

Bachevalier J, Mishkin M (1986) Visual recognition impairment follows ventromedial but not dorsolateral prefrontal lesions in monkeys. Behav Brain Res 20:249-261.

Barbas H, de Olmos J (1990) Projections from the amygdala to basoventral and mediodorsal prefrontal regions in the rhesus monkey. J Comp Neurol 300:549-571.

Buchanan SL (1991) Differential and reversal Pavlovian conditioning in rabbits with mediodorsal thalamic lesions: assessment of heart rate and eyeblink responses. Exp Brain Res 86:174-181.

Buchanan SL, Powell DA (1980) Divergencies in Pavlovian conditioned heart rate and eyeblink responses produced by hippocampectomy in the rabbit. Behav Neural Biol 30:20-38.

Buchanan SL, Powell DA (1982) Cingulate cortex: its role in Pavlovian conditioning. J Comp Physiol Psychol 96:755-774.

Buchanan SL, Powell DA (1993) Cingulothalamic and prefrontal control of autonomic function. In: Neurobiology of cingulate cortex and limbic thalamus (Vogt BA, Gabriel M, eds), pp 381-414. Cambridge, MA: Birkhauser.

Buchanan SL, Penney J, Powell DA (1994a) Lesions of the limbic thalamus differentially impair Pavlovian eyeblink conditioning under nonoptimal parametric conditions. Soc Neurosci Abstr 20:797.

Buchanan SL, Thompson RH, Maxwell BL, Powell DA (1994b) Efferent connections of the medial prefrontal cortex in the rabbit. Exp Brain Res 100:469-483.

Chachich ME, Powell DA (1992) Lesions of the medial prefrontal cortex impair reversal of classical conditioned eyeblink responses. Soc Neurosci Abstr 18:338.

Chachich ME, Powell DA (1994) Lesions centered on the central nucleus of the amygdala have no effect on discrimination or reversal of the Pavlovian conditioned eyeblink response. Soc Neurosci Abstr 20:797.

Clugnet M, LeDoux JE, Morrison SF (1990) Unit responses evoked in the amygdala and striatum by electrical stimulation of the medial geniculate body. J Neurosci 10:1055-1061.

Damasio AR (1994) Descartes error-emotion, reason and the human brain. New York: Putnam.

Damasio AR, Tranel D, Damasio H (1990) Individuals with sociopathic behavior caused by frontal damage fail to respond autonomically to social stimuli. Behav Brain Res 41:81-94.

Davis M (1992) The role of the amygdala in conditioned fear. In: The amygdala: neurobiological aspects of emotion, memory, and mental dysfunction (Aggleton JP, ed), pp 255-305. New York: Wiley.

Devinsky O, Morrell MJ, Vogt BA (1995) Contributions of anterior cingulate cortex to behaviour. Brain 118:279-306.

Frysztak RJ, Neafsey EJ (1994) The effect of medial frontal cortex lesions on cardiovascular conditioned emotional responses in the rat. Brain Res 643:181-193.

Fuster JM (1989) The prefrontal cortex: anatomy, physiology and neuropsychology of the frontal lobe, 2nd Ed. New York: Raven.

Gaffan D, Murray EA (1990) Amygdalar interaction with the mediodorsal nucleus of the thalamus and the ventromedial prefrontal cortex in stimulus-reward associative learning in the monkey. J Neurosci 10:3479-3493.

Ghelarducci B, Sebastiani L (1996) Contribution of the cerebellar vermis to cardiovascular control. J Auton Nerv Syst 56:149-156.

Gibbs CM, Powell DA (1988) Neuronal correlates of classically conditioned bradycardia in the rabbit: studies of the medial prefrontal cortex. Brain Res 442:86-96.

Gibbs CM, Powell DA (1991) Single-unit activity in the dorsomedial prefrontal cortex during the expression of discriminative bradycardia in rabbits. Behav Brain Res 43:79-92.
Gibbs CM, Prescott LB, Powell DA (1992) A comparison of multipleunit activity in the medial prefrontal and agranular insular cortices during Pavlovian heart rate conditioning in rabbits. Exp Brain Res 89:599-610.

Goldman-Rakic PS (1990) Cellular and circuit basis of working memory in prefrontal cortex of nonhuman primates. In: The prefrontal cortex: its structure, function and pathology, progress in brain research, Vol 85 (Uylings HBM, Van Eden CG, De Bruin JPC, Corner MA, Feenstra MGP, eds), pp 325-336. Amsterdam: Elsevier.

Gormezano I (1966) Classical conditioning. In: Experimental methods and instrumentation in psychology (Sidowski JB, ed), pp 385-420. New York: McGraw Hill.

Gormezano I, Gibbs CM (1988) Transduction of the rabbit's nictitating membrane response. Behav Res Methods Instrum \& Comput 20:18-21.

Greenhouse SW, Geisser S (1959) On methods in the analysis of profile data. Psychometrika 24:95-112.

Joseph JA, Powell DA (1980) Peripheral 6-hydroxydopamine administration in the rabbit (oryctolagus cuniculus): effects on Pavlovian conditioning. J Comp Physiol Psychol 94:1114-1125.

Kapp BS, Frysinger RC, Gallagher M, Haselton JR (1979) Amygdala central nucleus lesions: effect on heart rate conditioning in the rabbit. Physiol \& Behav 23:1109-1117.

Kapp BS, Wilson A, Pascoe JP, Supple W, Whalen PJ (1991) A neuroanatomical systems analysis of conditioned bradycardia in the rabbit. In: Neurocomputation and learning: foundations of adaptive networks (Gabriel M, Moore JW, eds), pp 53-90. Cambridge, MA: MIT.

Kazis E, Milligan WL, Powell DA (1973) Autonomic-somatic relationships: blockade of heart rate and corneoretinal potential. J Comp Physiol Psychol 84:98-110.

Kolb B (1984) Functions of the frontal cortex of the rat: a comparative review. Brain Res Rev 8:65-98.

Lacey BC, Lacey JI (1974) Studies of heart rate and other bodily processes in sensorimotor behavior. In: Cardiovascular psychophysiology: current issues in response mechanisms, biofeedback, and methodology (Obrist PA, Black AH, Brener J, DiCara LV, eds), pp 538-564. Chicago: Aldine.

LeDoux JE (1994) Emotion, memory and the brain. Sci Am 270:50-57. LeDoux JE, Sakaguchi A, Iwata J, Reis DJ (1986) Interruption of projections from the medial geniculate body to an arch-neostriatal field disrupts the classical conditioning of emotional responses to acoustic stimuli. Neuroscience 17:615-627.

Maxwell B, Powell DA, Buchanan SL (1994) Multiple and single unit activity in area 32 (prelimbic region) of the medial prefrontal cortex during Pavlovian heart rate conditioning in the rabbit. Cereb Cortex 4:230-246.

McCabe PM, Gentile CG, Markgraf CG, Teich AH, Schneiderman N (1992) Ibotenic acid lesions in the amygdala central nucleus but not in the lateral subthalamic area prevent the acquisition of differential Pavlovian conditioning of bradycardia in rabbits. Brain Res 580:155-163.

McDonald AJ (1987) Organization of amygdaloid projections to the mediodorsal thalamus and prefrontal cortex: a fluorescence retrograde transport study in the rat. J Comp Neurol 262:46-58.

McEchron MD, McCabe PM, Green EJ, Llabre MM, Schneiderman N (1991) Air puff versus shock unconditioned stimuli in rabbit heart rate conditioning. Physiol \& Behav 51:195-199.

Moyer Jr JR, Deyo RA, Disterhoft JF (1990) Hippocampectomy disrupts trace eye-blink conditioning in rabbits. Behav Neurosci 104:243-252.

Neafsey EJ, Hurley-Guis KM, Arvanitis D (1986) The topographical organization of neurons in the rat medial frontal, insular and olfactory cortex projecting to the solitary nucleus, olfactory bulb, periaqueductal gray and superior colliculus. Brain Res 377:261-270.

Parent MB, Quirarte GL, Cahill L, McGaugh JL (1995) Spared retention of inhibitory avoidance learning after posttraining amygdala lesions. Behav Neurosci 109:803-807.

Pascoe JP, Kapp BS (1985) Electrophysiological characteristics of amygdaloid central nucleus neurons during Pavlovian fear conditioning in the rabbit. Behav Brain Res 16:117-133.

Porrino LJ, Crane AM, Goldman-Rakic PS (1981) Direct and indirect pathways from the amygdala to the frontal lobe in rhesus monkeys. J Comp Neurol 198:121-136.

Posner MI, Petersen SE, Fox PT, Raichle ME (1988) Localization of cognitive operations in the human brain. Science 240:1627-1631.

Powell DA, Joseph JA (1974) Autonomic-somatic interaction and hippocampal theta activity. J Comp Physiol Psychol 87:978-986. 
Powell DA, Kazis E (1976) Blood pressure and heart rate changes accompanying classical eyeblink conditioning in the rabbit (Oryctolagus cuniculus). Psychophysiology 13:441-447.

Powell DA, Levine-Bryce D (1988) A comparison of two model systems of associative learning: heart rate and eyeblink conditioning in the rabbit. Psychophysiology 25:672-682.

Powell DA, Milligan WL (1975) Effects of partial and continuous reinforcement on conditioned heart rate and corneoretinal potential responses in the rabbit (Oryctolagus cuniculus). Psychol Rec 25:419-426.

Powell DA, Lipkin M, Milligan ML (1974) Concomitant changes in classically conditioned heart rate and corneoretinal potential discrimination in the rabbit (Oryctolagus cuniculus). Learn Motiv 5:532-547.

Powell DA, Watson KL, Buchanan SL (1990) Neuronal activity in the mediodorsal and intralaminar nuclei of the dorsal thalamus during classical heart rate conditioning. Brain Res 532:211-221.

Powell DA, Gibbs CM, Maxwell B, Levine-Bryce D (1993) On the generality of conditioned bradycardia in rabbits: assessment of CS and US modality. Anim Learn Behav 21:303-313.

Powell DA, Watson K, Maxwell B (1994) Involvement of subdivisions of the medial prefrontal cortex in learned cardiac adjustments. Behav Neurosci 108:294-307.

Powell G (1979) Brain and personality, pp 44-74. New York: Praeger.

Rosene DL, Roy NJ, Davis BJ (1986) A cryoprotection method that facilitates cutting frozen sections of whole monkey brains for histolog- ical and histochemical processing without freezing artifact. J Histochem Cytochem 34:1301-1315.

Schneiderman N, Gormezano I (1964) Conditioning of the nictitating membrane of the rabbit as a function of CS-US interval. J Comp Physiol Psychol 57:188-195.

Schneiderman N, Smith MC, Smith AC, Gormezano I (1966) Heart rate classical conditioning in rabbits. Psychon Sci 6:241-242.

Schwaber JS, Kapp BS, Higgins GA, Rapp PR (1982) Amygdaloid and basal forebrain direct connections with the nucleus of the solitary tract and the dorsal motor nucleus. J Neurosci 2:1424-1438.

Sebastiani L, La Noce A, Paton JFR, Ghelarducci B (1992) Influence of the cerebellar posterior vermis on the acquisition of the classically conditioned bradycardic response in the rabbit. Exp Brain Res 88:193-198.

Supple Jr WF, Kapp BS (1993) The anterior cerebellar vermis: essential involvement in classically conditioned bradycardia in the rabbit. J Neurosci 13:3705-3711.

Supple Jr WF, Leaton RN (1990) Cerebellar vermis: essential for classically conditioned bradycardia in rats. Brain Res 509:17-23.

Thompson RF (1991) Are memory traces localized or distributed? Neuropsychologia 29:571-582.

VanDercar DH, Schneiderman N (1967) Interstimulus interval functions in different response systems during classical discrimination conditioning of rabbits. Psychon Sci 9:9-10. 